\title{
Germanica
}

\section{La guerre - un jeu de l'esprit : à propos des Pensées de guerre (1914) de Thomas Mann}

Der Krieg - ein Gedankenspiel : zu Thomas Manns Gedanken im Kriege (1914)

Joëlle Stoupy

\section{OpenEdition}

\section{Journals}

Édition électronique

URL : http://journals.openedition.org/germanica/2236

DOI : 10.4000/germanica.2236

ISSN : 2107-0784

Éditeur

Université de Lille

\section{Édition imprimée}

Date de publication : 30 juin 2001

Pagination : 45-59

ISBN : 9782913857056

ISSN : 0984-2632

Référence électronique

Joëlle Stoupy, «La guerre - un jeu de l'esprit : à propos des Pensées de guerre (1914) de Thomas

Mann », Germanica [En ligne], 28 | 2001, mis en ligne le 04 octobre 2013, consulté le 06 octobre 2020.

URL : http://journals.openedition.org/germanica/2236; DOI : https://doi.org/10.4000/germanica.2236

Ce document a été généré automatiquement le 6 octobre 2020.

(c) Tous droits réservés 


\title{
La guerre - un jeu de l'esprit : à propos des Pensées de guerre (1914) de Thomas Mann
}

Der Krieg - ein Gedankenspiel : zu Thomas Manns Gedanken im Kriege (1914)

\author{
Joëlle Stoupy
}

1 Le texte Pensées de guerre que Thomas Mann rédige au tout début de la Première Guerre mondiale, en août et septembre 1914 et qui paraît en novembre de la même année dans la Neue Rundschau, documente de façon explicite l'enthousiasme qui s'empare de l'auteur à l'annonce de la guerre. Il permet de rapprocher sa réaction de celle de nombreux écrivains de sa génération, qui, comme Gerhart Hauptmann, Richard Dehmel, Hermann Hesse, Frank Wedekind, Hugo von Hofmannsthal ou Rainer Maria Rilke, se sont enflammés à l'idée d'une guerre susceptible de rompre la monotonie de leur époque et d'apporter une nouvelle vitalité face à une société ressentie comme décadente. Cet article marque aussi le début d'une série d'écrits inspirés de la guerre ${ }^{1}$. Ils aboutiront au bilan des Considérations d'un apolitique (1918) qui, pendant toute la durée des hostilités, éloigne Thomas Mann de son œuvre romanesque ; en 1914, il a, en effet, déjà commencé la rédaction de la Montagne magique (1924) qu'il interrompt alors pour se consacrer aux exigences du moment. De nombreuses lettres de l'auteur évoquent, à ce sujet, le besoin qu'il dit avoir ressenti, à la nouvelle de la guerre, de prêter main forte par ses écrits à la cause et à la culture allemandes qu'il voit menacées par les démocraties occidentales et de prendre parti pour l'Allemagne et la guerre. Avec une ardeur inattendue de la part d'un auteur déjà célèbre pour son regard ironique, Thomas Mann y fait l'éloge du militarisme de l'Allemagne dans lequel il reconnaît un garant de rigueur morale et de renouveau culturel.

2 Thomas Mann a relevé à diverses reprises le caractère improvisé de cet essai ${ }^{2}$, lui accordant ainsi peu d'importance sur le plan artistique et ne l'incluant après 1915 dans aucun projet d'édition. Cet article représente cependant une étape non négligeable dans l'évolution littéraire de son auteur. En effet, ce texte le confronte, pour la première fois, à des problèmes qui, de par leur actualité brûlante, dépassent ses 
préoccupations d'artiste et va le mener dans les années suivantes et presque contre ses propres convictions, à prendre en compte la réalité politique et à tenter de se positionner par rapport à elle. L'attitude de l'auteur des Pensées de guerre ressemble à celle du fils du marchand dans le Conte de la $672^{e}$ nuit (1895) de Hugo von Hofmannsthal. Le fils du marchand s'est longtemps complu dans le monde protégé de l'esthétisme, mais, un jour, précisément pour défendre cette idylle, il se voit contraint de sortir de ses retranchements d'esthète et d'affronter le monde extérieur auquel il avait toujours voulu échapper. Cette confrontation aura, dans le cas de Thomas Mann, une issue positive. En le conduisant aux Considérations d'un apolitique, qui, contrairement à ce que suggère le titre, ne sont pas un témoignage strictement apolitique, elle l'ouvre au domaine jusque-là négligé de la politique et lui fera peu à peu comprendre la nécessité de s'y intéresser et de prendre position.

$\mathrm{Si}$, en abordant le thème de la guerre, les Pensées de guerre abordent un sujet dépassant de simples considérations d'esthète, elles dissimulent cependant un discours esthétique où la guerre reste un prétexte. En véhiculant des concepts propres à l'œuvre narrative, reprenant notamment des idées de Tonio Kröger (1903) et de La Mort à Venise (1912), cet essai sert ainsi de charnière entre l'œuvre d'avant-guerre et celle d'après-guerre.

Comme de nombreux textes de l'époque, les Pensées de guerre témoignent, de façon précise, de l'engouement pour la guerre et les bouleversements qu'elle était susceptible d'amener. Le sentiment d'allégresse qui s'est emparé de la génération de l'auteur dans les premiers mois de guerre y est décrit sans équivoque. Thomas Mann y évoque les cœurs embrasés à l'idée de la guerre, supposée mettre fin à un monde décadent et annoncer le début d'une ère plus vigoureuse, d'une régénération :

Wie die Herzen der Dichter sogleich in Flammen standen, als jetzt Krieg wurde! Und sie hatten den Frieden zu lieben geglaubt, sie hatten ihn wirklich geliebt, ein jeder nach seiner Menschlichkeit, der eine auf Bauernart, der andere aus Sanftmut und deutscher Bildung. Nun sangen sie wie im Wettstreit den Krieg, frohlockend, mit tief aufquellendem Jauchzen - als hätte ihnen und dem Volke, dessen Stimme sie sind, in aller Welt nichts Besseres, Schöneres, Glücklicheres widerfahren können, als daß eine verzweifelte Übermacht von Feindschaft sich endlich gegen dies Volk erhob ${ }^{3}$;

5 Thomas Mann reconnaît la même réaction d'enthousiasme parmi les intellectuels les plus chevronnés et les gens les plus simples. La guerre est ressentie unanimement comme une promesse de félicité. Elle est l'orage purificateur qui met fin au sentiment latent de lassitude, un grand espoir, longtemps attendu, enfin réalisé. L'auteur parle certes de "catastrophe européenne $»^{4}$, mais sans y accorder beaucoup d'importance. C'est bien davantage le sentiment d'une joie intense qui prédomine dans sa description de l'annonce de la guerre; des jours qu'il nomme «inoubliables $»^{5}$, comme l'arrivée d'un bonheur inespéré. La guerre est décrite comme une force élémentaire, regorgeant de vie, une puissance supérieure à la raison, façonnant irrévocablement le destin des hommes :

Krieg! Es war Reinigung, Befreiung, was wir empfanden, und eine ungeheuere Hoffnung. Hiervon sagten die Dichter, nur hiervon. Was ist ihnen Imperium, was Handelsherrschaft, was überhaupt der Sieg? Unsere Siege, die Siege Deutschlands mögen sie uns auch die Tränen in die Augen treiben und uns nachts vor Glück nicht schlafen lassen, so sind doch nicht sie bisher besungen worden, man achte darauf, 
es gab noch kein Siegeslied. Was die Dichter begeisterte, war der Krieg an sich selbst, als Heimsuchung, als sittliche Not (Ess I, 193).

En servant, sans vraiment le vouloir, la propagande impérialiste de l'Allemagne, Thomas Mann prend ainsi des allures d'idéologue de la guerre. Ce n'est pas à son sens politique, peu développé encore à cette époque, remarque-t-il, qu'il attribue le fait d'avoir pressenti le caractère inévitable de la guerre. Selon lui, la guerre n'est pas mue par le goût de l'aventure, le besoin intense de sensations fortes, ce qui d'ailleurs ne serait qu'un désir de décadent, un besoin impérieux de narcotique. La guerre n'est pas non plus pour le Thomas Mann des Pensées de guerre l'expression d'une volonté impérialiste, mais elle est de façon prioritaire l'émanation d'une détermination morale, puisqu'elle doit permettre de sonner le glas d'une époque décadente. Comme le remarquait déjà Nietzsche dans Le Crépuscule des idoles (1888), l'homme de la fin du XIX siècle est un décadent, « un chaos [...], un soupir nihiliste, une confusion où l'on ne sait où donner de la tête ${ }^{6}$. Face à la multitude des savoirs et des impressions, il ne trouve plus en lui la volonté d'affirmer ou de nier. En s'inspirant de la critique nietzschéenne, Thomas Mann décrit lui aussi l'époque précédant la Première Guerre mondiale comme une période de confusion extrême et de pertes de valeurs. Le monde que la guerre emporte avec elle est considéré comme malade, car rongé par l'intellectualisme, comme morbide, car civilisé à l'excès ${ }^{7}$ :

Ist es zu viel gesagt, daß es kein Kriterium des Echten, nicht Mut noch Möglichkeit zur Verdammung mehr gab, daß buchstäblich niemand mehr aus noch ein wußte?

(Ess I, 193)

7 Pour Thomas Mann, la guerre a par contre donné lieu à un "radicalisme de la détermination $»^{8}$. Elle est l'épreuve suprême qui révèlera la supériorité morale de l'Allemagne, son énergie, "le mélange invincible d'activité et de persévérance ${ }^{9}$ que l'âme allemande doit à Frédéric II - personnage historique qui sert à étayer l'idéologie de l'auteur - et dont l'Allemagne sortira grandie ; son militarisme étant, selon l'auteur, non pas une preuve de barbarie, mais une marque indéniable du bon fonctionnement de ses institutions. Ainsi, la guerre doit permettre l'éclosion de vertus indispensables à la grandeur d'une nation:

Deutschlands ganze Tugend und Schönheit - wir sahen es jetzt - entfaltet sich erst im Kriege. Der Friede steht ihm nicht immer gut zu Gesicht - man konnte im Frieden zuweilen vergessen, wie schön es ist. (Ess I, 199).

8 Si certains contemporains ont partagé ces idées, comme l'atteste par exemple la réaction d'enthousiasme de Richard Dehmel à la lecture des Pensées de guerre, alors qu'il se trouve lui-même au front ${ }^{10}$, - ces idées nous apparaissent aujourd'hui comme de la "propagande poussiéreuse $~^{11}$. Mais les Pensées de guerre ne contiennent, qu'à première vue, une réflexion sur la guerre en tant que telle. Une lecture plus approfondie montre que l'essence de ce texte est tout d'abord esthétique. La guerre se révèle être un jeu de l'esprit au service de préoccupations artistiques. Elle offre, en outre, à Thomas Mann une solution toute particulière à des problèmes d'ordre privé: au problème de l'homosexualité et de sa tentative de légitimation.

Quelques mois avant le début des hostilités, à la fin de l'année 1913, dans une lettre à son frère Heinrich datant du 8 novembre, Thomas Mann tire un bilan de sa carrière artistique qui est empreint de doute. Il ne peut s'empêcher de mettre en avant la 
sensation d'avoir fait son temps ${ }^{12}$, de s'être trop longtemps complu dans la description et l'analyse de la décadence, son terrain de prédilection depuis les nouvelles de jeunesse, de ne pas être en accord avec les exigences du nouveau siècle, de ne pas avoir su se positionner face aux défis d'une société en mutation, comme son frère selon lui a su le faire. Lorsque la guerre éclate en 1914, Thomas Mann va alors rapidement la saluer comme salvatrice, car il y découvre une issue possible aux problèmes artistiques qui sont les siens. Paradoxalement, la guerre est supposée l'aider à retrouver le sentiment de vie, d'immédiateté et de vitalité qui manque à l'artiste décadent.

Les lettres à Heinrich Mann, écrites au tout début de la guerre, laissent entrevoir l'opportunité que décèle peu à peu Thomas Mann dans les bouleversements qui s'imposent à l'Europe. À l'annonce de la guerre, l'auteur apparait tout d'abord, dans une lettre du 30 juillet 1914, "ébranlé et confus à cause de l'horrible poids de la réalité $\aleph^{13}$. Supposée apporter un changement tangible dans sa vie, la guerre est ressentie comme une fatalité dépassant ses propres forces, un événement incontournable contre lequel il ne peut rien ; elle est « l'effroyable $»^{14}$. Il ne la salue pas ouvertement encore à cette date, mais ne s'y oppose pas catégoriquement. Son attitude face à la guerre devient plus explicite dans une lettre rédigée le 7 août 1914. Il confie à son frère s'être laissé surprendre et être pris dans un rêve. La guerre prend ici déjà les traits du «dieu étranger " qui afflige le petit monsieur Friedemann et Gustav von Aschenbach et qui détruira leur existence ascétique et bourgeoise construite à force de courage et de patience ${ }^{15}$. Thomas Mann, d'ordinaire si attaché à un certain confort, envisage alors sans crainte d'être ruiné par la guerre et se laisse griser par les changements qu'elle apportera :

In Gottes Namen! Was will das besagen gegen die Umwälzungen, namentlich die seelischen, die solche Ereignisse im Großen zur Folge haben müssen! Muß man nicht dankbar sein für das vollkommen Unerwartete, so große Dinge erleben zu dürfen $?^{16}$

11 Dans une lettre du 18 septembre 1914, le camp qu'il a choisi est à présent tout à fait défini. Il fête l'arrivée de la guerre et la qualifie de "grande guerre populaire foncièrement honnête et solennelle $»^{17}$.

12 S'il la salue avec autant de conviction, ce n'est cependant pas, en premier lieu, par patriotisme, même si l'auteur le dit à plusieurs reprises ${ }^{18}$. Comme nous l'avons dit, ses Pensées de guerre sont tout d'abord des pensées d'esthète. Il faut, en fait, remonter jusqu'à l'œuvre de jeunesse de Thomas Mann pour comprendre que la solution qu'incarne la guerre à son dilemme d'artiste découle d'une réflexion entamée au début de son œuvre, et qu'il n'existe pas vraiment de rupture entre l'auteur des Buddenbrook et celui des Pensées de guerre ${ }^{19}$. La guerre ne fait qu'apporter à Thomas Mann une réponse au problème de la décadence et plus particulièrement à celui du dilettantisme de la fin de siècle ${ }^{20}$ auquel il a été confronté dans sa jeunesse, comme la plupart des auteurs de sa génération, influencés notamment par la critique de théoriciens de la décadence, tels que Friedrich Nietzsche et Paul Bourget. Le texte Pensées de guerre expérimente ainsi avec le surpassement du dilettantisme - tout en restant profondément ancré dans une attitude dilettante et définit la nouvelle esthétique de son auteur, comme avant lui, les notes sur Geist und Kunst (1912) ont tenté de le faire et sur lesquelles il s'appuie ici.

13 «Ce siècle qui finit est plein de dilettantes... $»^{21}$ lit-on dans un article d'Henri Becques en 1884. À la même époque, Paul Bourget (1852-1935), auteur psychologique à la mode 
et théoricien de la décadence fort apprécié dans les années 1890 en Europe, définit le premier l'attitude du dilettantisme dans son essai sur Ernest Renan :

C'est beaucoup moins une doctrine qu'une disposition de l'esprit, très intelligente à la fois et très voluptueuse, qui nous incline tour à tour vers les formes diverses de la vie et nous conduit à nous prêter à toutes ces formes sans nous donner à aucune ${ }^{22}$.

Le dilettantisme, attitude de détachement face à la vie et aux idées, est reconnu par de nombreux jeunes écrivains de langue allemande des années 1890 comme étant une des caractéristiques de l'artiste moderne. Il est le produit d'une époque qui aime comprendre et jouer avec les idées : «Le bien et le mal, la douleur et la joie, tout est matière pour vous à ce jeu de votre esprit $»^{23}$, reproche le marquis de Montfanon au jeune écrivain Dorsenne dans le roman de Bourget Cosmopolis (1893), car celui-ci se prête aux sentiments et aux idées sans pouvoir s'engager concrètement, sans pouvoir se « donner ». Comme le montrent Nietzsche et Bourget, l'époque de la fin de siècle est affaiblie notamment par un abus de réflexion: «Nous sommes malades d'un excès de pensée critique, malades de trop de littérature, malades de trop de science $\aleph^{24}$ dit l'essai de Bourget sur Alexandre Dumas fils. Ainsi, « l'extrême civilisation » qui a trop cultivé l'intelligence au détriment de forces inconscientes est ressentie, non pas comme un apogée, mais comme une décadence. Les conséquences du dilettantisme sont nombreuses: perte d'immédiateté, d'unité et de sens moral, affaiblissement de la volonté et perte d'engagement et d'action :

La haute société contemporaine [...] est parvenue à cette heure, coupable peut-être, à coup sûr délicieuse, où le dilettantisme remplace l'action ; [...] C'est probablement une loi que les sociétés barbares tendent de toutes leurs forces à un état de conscience qu'elles décorent du titre de civilisation, et qu'à peine cette conscience atteinte la puissance de vie tarisse en elles. Les Orientaux disent souvent : Quand la maison est prête la mort entre... ${ }^{25}$

Le désir de dépasser le dilettantisme a donné lieu, vers 1900, à la nécessité d'un retour vers une affirmation de la vie, de l'action, à la recherche de détermination morale allant à l'encontre de l'intellectualisme et du relativisme de la fin de siècle. Ce besoin d'engagement découle directement de la nécessité d'échapper au dilettantisme, à la fascination ouverte à toutes les idées qui exclut tout engagement en faisant de l'homme un spectateur de la vie. C'est au "Nietzsche triumphans ${ }^{26}$ qu'appartient l'avenir. Comme le remarque Thomas Mann dans une note de Geist und Kunst, alors que sa propre génération était captivée par tout ce qui était pathologique, la nouvelle génération cherche, en ce début du $x^{e}$ siècle, à cultiver «tout ce qui, d'une façon ou d'une autre, est sain en nous $»^{27}$.

Comme nous le savons aujourd'hui, Thomas Mann connaissait bien le personnage du dilettante ${ }^{28}$. Il parcourt ses œuvres de jeunesse et ses personnages en possèdent certains traits: Paillasse, Christian, Thomas et Hanno Buddenbrook, Tonio Kröger et Gustav von Aschenbach connaissent ou ont connu par exemple le danger de l'introspection et de l'analyse qui paralyse l'action. Quelques années seulement avant la guerre, Thomas Mann replace encore la carrière littéraire de Gustav von Aschenbach dans La Mort à Venise (1912), irréfutablement dans les courants littéraires de la fin de siècle, ceux auxquels l'auteur lui-même a été confronté. De multiples interprétations ont d'ailleurs relevé très tôt l'appartenance de cette nouvelle à l'esthétique décadente, en suivant notamment et à juste titre l'auteur lui-même qui voyait dans La Mort à Venise «la réalisation moralement et formellement la plus poussée et la plus concise du problème de la décadence et de celui de l'artiste $»^{29}$. Nous savons aussi que Thomas 
Mann reconnaissait avoir conçu Aschenbach comme un parent des héros de son œuvre de jeunesse et qu'il est justifié de le rapprocher par exemple d'un personnage décadent tel que Thomas Buddenbrook ${ }^{30}$. Thomas Buddenbrook a, lui aussi, tenté d'endiguer son penchant pour la décadence, se résumant notamment en une trop grande réflexivité, par un surcroît de volonté. Il a conscience que l'auto-analyse excessive tue les forces vitales et l'action, alors que ses ancêtres connaissaient, eux, la spontanéité et l'immédiateté. Mais il ne parvient pas à faire preuve d'une détermination semblable à la leur et deviendra partiellement victime de la dangereuse "maladie de la volonté ». Comme Thomas Buddenbrook, Gustav von Aschenbach est l'héritier de l'époque décadente et sa volonté n'est qu'un tour de force pour échapper à son attirance pour le " gouffre ", un combat permanent avec « cette fatigue grandissante dont nul ne devait avoir connaissance $»^{31}$.

Nous savons que Thomas Mann connaissait le type du dilettante aussi pour avoir copié dans ses notes une partie de la préface du Disciple de Paul Bourget ${ }^{32}$. Thomas Mann connaissait le personnage du dilettante Julien Dorsenne, tiré du roman de Paul Bourget Cosmopolis. En 1896, il débute son article Ein nationaler Dichter en s'arrêtant sur celui-ci et sur la critique qu'en fait son adversaire dans le roman, le légitimiste Montfanon. Julien Dorsenne, écrivain de renom dans le roman de Bourget, ne se préoccupe pas de prendre position: "Je ne me pique pas de juger la vie, moi, Monsieur le ligueur", répond-il à Montfanon, "j'aime à la regarder et à la comprendre " ${ }^{33}$. L'écrivain Gustav von Aschenbach, lui aussi, s'est voué dans sa jeunesse à l'esprit d'analyse, au psychologisme, au besoin de comprendre pour comprendre : «Il avait sacrifié à l'esprit, abusivement exploité la connaissance, broyé le grain de semence $[. ..] »^{34}$. Mais à la recherche d'une "fermeté morale », déterminé à " renier le savoir [...] pour peu que ce savoir soit en rien susceptible de paralyser, de décourager, d'avilir la volonté, l'action, le sentiment et la passion elle-même $\aleph^{35}$, Aschenbach a essayé d'atteindre un équilibre. Sa nouvelle orientation conçue comme "un accès de dégoût face au psychologisme indécent de l'époque $»^{36}$ - indécent parce qu'il rend difficile tout jugement moral à celui qui ne veut que comprendre sans juger - signifiait «l'abandon de toute incertitude morale, de toute sympathie pour le gouffre, le renoncement au laisser-aller qu'implique cette maxime dictée par la compassion, selon laquelle tout comprendre veut dire tout pardonner $\aleph^{37}$. Les œuvres de maturité d'Aschenbach revendiquent ainsi une portée morale et découlent directement d'une opposition au psychologisme et au dilettantisme. Son œuvre Ein Elender indique que la fermeté morale est à nouveau possible, son roman Maja est, contrairement aux romans psychologiques, riche en personnages et montre l'intérêt qu'Aschenbach porte à présent, non plus à l'analyse du moi, mais au monde extérieur ${ }^{38}$. L'étude de la carrière littéraire de ce personnage, très proche de celle de Thomas Mann lui-même, nous permet ainsi de retrouver dans La Mort à Venise, l'ambition de surmonter la décadence et son «inconvenant psychologisme", son dilettantisme. Nous retrouvons dans la conception de l'art d'Aschenbach que Thomas Mann voue cependant à l'échec, l'itinéraire allant du dilettantisme dépourvu de préoccupation morale, qui ne veut que comprendre, sans porter de jugement de valeur, à une fermeté morale au-delà du savoir. Ce besoin découle de la nécessité d'échapper au dilettantisme, à la fascination pour une pensée ouverte à toutes les connaissances et aux contradictions du moi, qui ne pouvait plus étreindre de certitudes et excluait donc l'engagement, en faisant de l'homme un spectateur de la vie, un dilettante. 
Si dans La Mort à Venise Gustav von Aschenbach échoue dans sa tentative d'échapper à la décadence, Thomas Mann prétend lui, par le biais de la guerre, réussir ce tour de force. Sa nouvelle attitude semble lui permettre, en outre, d'apporter de nouvelles réponses à ses lecteurs et de rester ainsi représentatif de son époque. Il n'est pas étonnant alors de retrouver dans Pensées de guerre, presque mot pour mot, les ambitions énoncées dans La Mort à Venise qui, à présent, semblent réalisables à son auteur :

Eine sittliche Reaktion, ein moralisches Wieder-fest-werden hatte eingesetzt oder bereitete sich vor; ein neuer Wille, das Verworfene zu verwerfen, dem Abgrund die Sympathie zu kündigen, ein Wille zur Geradheit, Lauterkeit und Haltung wollte Gestalt werden (Ess I, 192).

La guerre devient alors une affaire morale, car elle prétend guérir de la décadence, permet un retour à l'action et à l'engagement en exigeant une prise de position, alors que le dilettantisme reniait tout parti pris. Thomas Mann dit d'ailleurs de son texte qu'il est une «action $»^{39}$, une décision d'agir et de vouloir, la maladie de la volonté étant la maladie du décadent par excellence.

La tentative de valoriser la guerre dans Pensées de guerre est aussi la tentative de justifier des choix d'ordre privé. Depuis son mariage avec Katia Pringsheim, en 1905, Thomas Mann a décidé de refouler ses tendances homosexuelles, de se "ranger ${ }^{40}$. Il a ainsi opté pour l'éthique bourgeoise, pour ce qui représente à ses yeux le monde paternel du devoir au détriment de ce qui symbolise pour l'auteur l'héritage maternel, «la tendance artistique et sensuelle $»^{41}$. Dans un essai sur Adelbert von Chamisso, écrit peu avant son voyage à Venise en 1911, Thomas Mann entrevoit, à travers le poète français et la décision de ce dernier de fonder une famille, de se fixer, un garant de réussite qui a permis à Chamisso de devenir un auteur classique; ce à quoi Thomas Mann prétend aussi. Mais dans La Mort à Venise, il montre alors que la préférence donnée au monde paternel, établi aux détriments de penchants profonds, est simplificateur et voué à l'échec. La guerre, par contre, semble offrir à l'auteur l'avantage de réussir la synthèse de deux mondes qui jusque-là s'étaient avérés antithétiques: du monde de l'esprit et de la vie, de l'intellectualisme et de l'irrationalisme, du monde bourgeois et artiste, de l'héritage paternel et maternel ${ }^{42}$. Dans Pensées de guerre, Thomas Mann présente ainsi la guerre comme l'arrivée de forces irrationnelles - le mot « Heimsuchung » revient plusieurs fois dans le texte et les lettres de cette période et rappelle le sentiment que connaît le petit monsieur Friedemann lorsqu'il voit Gerda von Rinnlingen ou Aschenbach Tadzio ${ }^{43}$. À travers la guerre, Thomas Mann entrevoit la possibilité de se laisser transporter, aux yeux de tous, par la force élémentaire qu'elle représente et qui ressemble, c'est lui-même qui le dit dans Pensées de guerre, à l'acte sexuel. Mais la guerre lui permet, d'un autre côté, de jouer un rôle comme être spirituel et d'établir une synthèse avec la vie. L'artiste ne touche-t-il pas par ses écrits les soldats dans les tranchées, comme le laissent supposer les lettres que Thomas Mann dit recevoir du front ${ }^{44}$; ainsi, la distance entre art et vie semble abolie, la synthèse longtemps recherchée apparamment atteinte. Thomas Mann peut se laisser envahir par une force élémentaire comparable à celle qui envahit Aschenbach à la vue de Tadzio; il voit l'ordre bourgeois se renverser, comme Aschenbach se délectait à l'idée d'une Venise dévastée, en accord avec sa vie intérieure. Mais contrairement à Aschenbach, Thomas Mann peut rester maître de son monde artistique, administrer sa renommée, comme le faisait Aschenbach au début de La Mort à Venise, goûter à la 
tentation de l'élémentaire, sans devoir s'y perdre, donner aussi bien le change au monde paternel, celui de l'ordre qu'au monde maternel, celui du chaos, satisfaire son désir de grandeur et de respectabilité sans perdre son penchant pour le « gouffre » en l'ayant sublimé. La guerre est ainsi décrite dans Pensées de guerre comme une fusion et une ivresse de se retrouver, porteuse de visions communautaires. Alors qu'après avoir fait la connaissance de Tadzio, Aschenbach ne s'inquiétait plus de dilapider son temps et sa force, sans aucun profit pour son art et que l'idée d'un retour à ses fonctions d'auteur célèbre le révulsait, Thomas Mann croit, par le biais de la guerre, pouvoir réussir là où Aschenbach a échoué.

21 L'antithèse art et vie qui parcourt les textes de jeunesse semble ainsi subitement, avec l'irruption de la guerre, s'accomplir dans une synthèse que Thomas Mann baptise à présent du terme de Kultur, emprunté à Nietzsche. Le concept antagoniste porte, lui, à présent le nom de Zivilisation. Ce concept, qui représente les puissances occidentales et sera au centre de la rhétorique des Considérations d'un apolitique, renferme pour l'auteur des Pensées de guerre, en premier lieu, l'esprit de décadence qu'il s'agit de surmonter. Comme le montre déjà le petit décadent Hanno Buddenbrook, l'idée de civilisation est à la fois culmination, mais aussi irrémédiablement déclin. Elle porte en elle un germe de mort, un besoin de conscience trop excessif qui détruit les forces vitales de l'homme. Pour le Thomas Mann de 1914 qui a décidé de tourner le dos à la décadence, il ne s'agit plus de se reconnaître dans la notion de civilisation, mais de se donner les moyens d'accomplir le rêve d'immédiateté dont parle Fiorenza (1905), ce «miracle de la naïveté renaissante $"^{45}$, nécessaire à l'auteur classique. Depuis le succès de Buddenbrook (1901), le parcours artistique de Thomas Mann est jalonné de tentatives plus ou moins malheureuses de retrouver ce sentiment d'immédiateté, de « vie ». Contrairement à ses espoirs, Fiorenza ne lui a pas apporté le succès escompté et a été jugé d'artificieux ; la synthèse entre art et vie, décrite dans son deuxième roman Altesse royale, a été, elle, perçue comme mensongère par son auteur lui-même. Le récit La Mort à Venise illustre, de son côté, l'échec d'un écrivain qui avait les prétentions d'un classique, mais se laisse emporter par l'irrationnel, sans pouvoir résister et rester maître de son art. L'artiste, moderne tout autant que classique, ne devra plus cultiver l'esprit d'analyse et d'observation propres à la décadence de façon outrancière. Il sera marqué par le désir de renouer avec les forces irrationnelles de l'art. À la recherche d'un nouveau classicisme, Thomas Mann désire en finir avec la sensation de n'être peut-être qu'un "littérateur" pour devenir une figure emblématique de son époque, mais aussi un classique à la mesure de Goethe. Dans Pensées de guerre, Goethe est ainsi représentatif de l'idée de Kultur, détenteur d'un pouvoir irrationnel et mystique qui lui fit par exemple ressentir le tremblement de terre de Messine grâce à des affinités secrètes avec la nature.

Thomas Mann projette ainsi sur le concept de Zivilisation tout ce qu'il ne veut plus être et opte pour celui de Kultur, qu'il dit propre à l'Allemagne. Les nations opposées à l'Allemagne représentent à ses yeux la civilisation décadente qui a voulu, de toutes ses forces, étouffer le côté instinctif et irrationnel dans l'homme et, en le civilisant et le rationalisant l'a affaibli, tandis que l'Allemagne a su, selon lui, conservé un potentiel de forces vitales qui l'oppose irréductiblement aux civilisations occidentales et la rend habile à la guerre. L'idée de Kultur exprime, par contre, une forme de société où règne certes l'ordre

Kultur ist Geschlossenheit, Stil, Form, Haltung, Geschmack, ist irgendeine gewisse geistige Organisation der Welt [...] (Ess I, 188), 
mais qui aurait conservé, d'un autre côté, des instincts riches, qui la rend apte à surpasser la décadence. Pensées de guerre retient donc de l'idée de Zivilisation non pas l'idée de démocratie, comme dans les Considérations d'un apolitique, mais tout d'abord l'oppression de l'instinct par la réflexion et le rationalisme. Elle est « l'ennemi juré des pulsions, des passions $»^{46}$ et ne laisse ainsi, aux yeux de l'auteur, aucune légitimité à ce qu'il appellera en 1924, dans une lettre à André Gide, « la région sentimentale qui, aussi selon mon opinion, ne peut être méprisée et condamnée que par le barbarisme [!] et l'ignorance [...] $»^{47}$, à l'homosexualité. La nouvelle esthétique de Thomas Mann tente par le biais de la guerre d'intégrer, par contre, dans une synthèse qui reste hasardeuse et forcée, l'élément instinctif, sensuel et « démoniaque » dans le but premier de trouver l'équilibre entre sensualité et esprit de l'art classique qu'il reconnaît par exemple en 1912 dans les Affinités électives de Goethe. politique restent encore étrangères à son auteur. Si elles contiennent le germe d'une nouvelle attitude, elles restent un témoignage d'esthétisme. La guerre y fait l'objet d'une construction mentale à certains égards brillante, mais terrifiante par les implications que peut avoir sa glorification. Elle demeure l'illusion d'un artiste manipulant la réalité sans vraiment l'atteindre. À aucun moment, semble-t-il, Thomas Mann ne prend peur des conséquences et inconséquences de sa pensée et de la défense de l'éclosion de forces irrationnelles. On est guère étonné d'entendre le pacifiste Romain Rolland s'insurger contre le "délire d'orgueil et de fanatisme irrité » et la «démence $»^{48}$ que contiennent les Pensées de guerre.

Une attitude ambivalente sous-tend ainsi l'approche qu'a Thomas Mann de la guerre dans ses Pensées de guerre. Nous sommes certes toujours confrontés à l'esthète dilettante qui ajuste un événement lourd de conséquences pour l'humanité - la guerre - aux besoins de son monde intérieur. Cet essai reste donc malgré tout un exercice de dilettantisme, un jeu de l'esprit, puisque la réalité de la guerre n'y trouve pas vraiment sa place. Mais, comme ce texte contient paradoxalement une première prise de position - en faveur de la guerre, il signifie aussi un premier abandon du besoin d'indépendance absolu de l'artiste. Cet essai est, par ailleurs, digne de retenir l'attention, car c'est à travers lui que se révèle explicitement pour le frère aîné de Thomas Mann, Heinrich, l'antagonisme déjà latent qui les sépare. Il marquera le début de leur querelle qui durera jusqu'en 1922. Celle-ci poussera Thomas Mann, dans les années vingt, à réajuster ses positions face au nationalisme, en faveur de l'idée de démocratie et d'un rapprochement européen. 


\section{NOTES}

1. Frédéric et la grande coalition (Friedrich und die große Koalition), rédigé entre septembre et décembre 1914, Bonnes nouvelles du Front (Gute Feldpost) entre septembre et octobre 1914, À la rédaction du "Svenska Dagbladet», Stockholm (Brief an die Zeitung "Svenska Dagbladet», Stockholm) entre avril et mai 1915, Pensées au sujet de la guerre (Gedanken zum Kriege) en juillet 1915 et $\mathrm{Au}$ journal de l'armée A.O. K. 10 (An die Armeezeitung A.O.K. 10) au printemps 1916.

2. "Und so entstand jene frühe und rasche Improvisation, die "Gedanken im Kriege" überschrieben war [...]» (Th. Mann: Betrachtungen eines Unpolitischen. Bd. XII, in: Th.M.: Gesammelte Werke in dreizehn Bänden, Frankfurt/M.: Fischer Taschenbuch Verlag, 1974, p. 161 [cette édition sera citée GW]). « Er [der Aufsatz] ist Journalistik, nichts weiter [...]» (Dichter über ihre Dichtungen. Thomas Mann. Teil I: 1889-1917. Herausgegeben von Hans Wysling unter Mitwirkung von Marianne Fischer, Zürich/München/Frankfurt/M. : Heimeran/S. Fischer, 1975, p. 595 [lettre à Hanna Rademacher, 30.11.1914]).

3. Th. Mann : Essays. Band 1: Frühlingssturm 1893-1918. Herausgegeben von Hermann Kurzke und Stephan Stachorski, Frankfurt/M : S. Fischer Verlag, 1993, p. 191 (cet ouvrage sera cité Ess I).

4. "Wir hatten an den Krieg nicht geglaubt, unsere politische Einsicht hatte nicht ausgereicht, die Notwendigkeit der europäischen Katastrophe zu erkennen. » (Ess I, 192).

5. «Erinnern wir uns des Anfangs - jener nie zu vergessenden ersten Tage, als das Große, das nicht mehr für möglich Gehaltene hereinbrach!» (Ibid.).

6. « Ein Chaos [...], ein nihilistisches Seufzen, ein Nicht-wissen-wo-aus-noch-ein » (Fr. Nietzsche : Götzendämmerung. Kritische Studienausgabe 6. Herausgegeben von Giorgio Colli und Mazzino Montinari, München : dtv / Berlin/New York : de Gruyter, 1988, p. 152.

7. «Wimmelte sie [diese Welt des Friedens] nicht von dem Ungeziefer des Geistes wie von Maden? Gor und stank sie nicht von den Zersetzungsstoffen der Zivilisation. » (Ibid.).

8. « Radikalismus der Entschlossenheit» (Ibid., p. 193).

9. « diese nie zu besiegende Mischung von Aktivität und durchhaltender Geduld» (Ibid., p. 194).

10. «Vorgestern las ich im Schützengraben Ihre Gedanken über den Krieg (Neue Rundschau). Ich muß sagen, daß mir jedes Wort aus der Seele gesprochen ist [...]. Diese stille Vorkämpfer-Arbeit ist vielleicht doch ersprießlicher für die Zukunft als aller Kriegslärm der Gegenwart. » (Th. Mann : Briefwechsel mit Autoren, hrsg. von Hans Wysling, FrankfurtMain : S. Fischer, 1988, p. 150 [lettre du 25.11.1914]).

11. «Der Rest der Kriegsgedanken ist verstaubte Propaganda, ohne Wahrheit und ohne tieferes Motiv, sorglos recherchiert, flüchtig geschrieben und schnell wieder vergessen.» (H. Kurzke : Thomas Mann. Epoche - Werk - Wirkung. 3., erneut überarb. Auflage, München : C.H. Beck, 1997, p. 139).

12. «Ich bin ausgedient, glaube ich, und hätte wahrscheinlich nie Schriftsteller werden dürfen. » (Thomas Mann - Heinrich Mann : Briefwechsel 1900-1945, hrsg. von Hans Wysling. Frankfurt/M. : Fischer Taschenbuch Verlag, 1995, p. 166).

13. «Ich muß sagen, daß ich mich erschüttert und beschämt fühle durch den furchtbaren Druck der Realität. » (Ibid., p. 169).

14. « Man ist zu civilen Gemütes um das Ungeheuerliche für möglich zu halten. » (Ibid.).

15. «Im Anfang, wie in der Mitte : Vom "Kleinen Herrn Friedemann" zum "Tod in Venedig”, der viel späteren Erzählung vom Kommen des "fremden Gottes" spannt sich der Bogen » (GW XIII, p. 136).

16. Thomas Mann - Heinrich Mann : Briefwechsel 1900-1945, op. cit., p. 170.

17. «Diesen großen, grundanständigen, ja feierlichen Volkskrieg » (Ibid., p. 172). 
18. Cf. lettre du 10.2.1915 à Albert Ehrenstein : «Aber es giebt Augenblicke, wo man sich gern und mit Leidenschaft kompromittiert, und so ist das Stückchen Journalistik, worauf Sie anspielen, dem heftigen Bedürfnis entsprungen, meinen bescheidenen Kopf einmal unmittelbar in den Dienst der deutschen Sache zu stellen, die mir groß und tief anständig erschien und die ich aus aller Welt mit Schimpf überschüttet sah.» (Dichter über ihre Dichtungen. Thomas Mann. Teil I: 1889-1917, op. cit., p. 596).

19. Contrairement à ce qu'affirme par exemple Louis Leibrich: "Mais sa prise de position constitue une erreur par rapport à ses orientations authentiques et une rupture avec son passé spirituel » (L. Leibrich : Thomas Mann. Une recherche spirituelle, Paris : Aubier, 1974, p. 103).

20. Cf. J. Stoupy : "Maître de l'heure». Die Rezeption Paul Bourgets in der deutschsprachigen Literatur um 1890, Frankfurt/M. : Lang, 1996, p. 42-46.

21. H. Becque, in Le Gaulois, 30 mai 1884.

22. P. Bourget : Essais de psychologie contemporaine, Paris : Lemerre, 1883, p. 59.

23. P. Bourget : Cosmopolis, Paris : Lemerre, 1893, p. 468.

24. P. Bourget: Nouveaux Essais de psychologie contemporaine, Paris : Lemerre, 1886, p. 62.

25. P. Bourget : Nouveaux Essais de psychologie contemporaine, Paris : Lemerre, 1886, p. 62.

26. "Geist und Kunst». Thomas Manns Notizen zu einem "Literatur-Essay». Ediert und kommentiert von Hans Wysling, in P.Scherrer/H. Wysling: Quellenkritische Studien zum Werk Thomas Manns. Thomas-Mann-Studien, Band 1, Bern/München : Francke Verlag, 1967, p. 144.

27. « Die Forderung der Zeit ist, alles, was irgend gesund ist in uns, zu kultivieren. » (Ibid., p. 142). 28. K. Schröter a remarqué le premier dans l'œuvre du jeune Thomas Mann les traces de la lecture de Paul Bourget (K. Schröter: Thomas Mann in Selbstzeugnissen und Bilddokumenten, Reinbek bei Hamburg : Rowohlt Taschenbuch Verlag, 1964, p. 31-44).

29. «Die moralisch und formal zugespitzteste und gesammeltste Gestaltung des Décadence- und Künstlerproblems » (GW XIII, p. 151).

30. «Ich schuf mir einen modernen Helden [...], einen Bruder Thomas Buddenbrooks [...]» (Ibid., p. 148).

31. «Zwar liebte er ihn und liebte auch fast schon den entnervenden, sich täglich erneuernden Kampf zwischen seinem zähen und stolzen, so oft erprobten Willen und dieser wachsenden Müdigkeit, von der niemand wissen [...] durfte» (GW VIII, p. 448).

32. Th. Mann: Notizbücher. 1-6. Herausgegeben von Hans Wysling und Yvonne Schmidlin. Frankfurt/Main, S. Fischer, 1991, p. 24.

33. P. Bourget: Cosmopolis, op. cit., p. 30.

34. «Er hatte dem Geiste gefrönt, mit der Erkenntnis Raubbau getrieben, Saatfrucht vermahlen [...] » (GW VIII, p. 454). «L'analyse, s'écriait-il lui-même, tue la spontanéité. Le grain moulu en farine ne saurait plus ni germer ni lever... " (nous soulignons) (P. Bourget: Nouveaux Essais, op. cit., p. 290).

35. «[...] das Wissen zu leugnen, es abzulehnen, erhobenen Hauptes darüber hinwegzugehen, sofern es den Willen, die Tat, das Gefühl und selbst die Leidenschaft im geringsten zu lähmen, zu entmutigen, zu entwürdigen geeignet ist. » (GW VIII, 454-455).

36. « Ausbruch des Ekels gegen den unanständigen Psychologismus der Zeit » (GW VIII, p. 455).

37. «Abkehr von allem moralischen Zweifelsinn, von jeder Sympathie mit dem Abgrund, die Absage an die Laxheit des Mitleidssatzes, daß alles verstehen alles verzeihen heiße [...] » (Ibid.).

38. Comme son personnage, Thomas Mann a tenté dans les Considérations d'un apolitique de se positionner parmi ceux qui, pour surmonter la décadence, ont renoncé au psychologisme : « Was mich betrifft, so habe ich mir an verschiedenen Punkten der nachfolgenden Aufzeichnungen deutlich zu machen gesucht, inwiefern ich mit dem Neuen zu tun habe, inwiefern auch in mir etwas ist von jener "Entschlossenheit", jener Absage an den "unanständigen Psychologismus" der abgelaufenen Epoche, an ihr laxes und formwidriges tout comprendre [...] » (GW XII, p. 28). 
39. «Meine kleine Aktion aber, als solche, ist gelungen» (Dichter über ihre Dichtungen. Thomas Mann. Teil I : 1889-1917, op., cit., p. 597).

40. «[...] und Du nennst mich gewiß einen feigen Bürger. Aber Du hast leicht reden. Du bist absolut. Ich dagegen habe geruht, mir eine Verfassung zu geben.» (Thomas Mann - Heinrich Mann : Briefwechsel 1900-1945, op. cit., p. 114).

41. GW XI, p. 98.

42. «Auf subtile Weise schien der Krieg sogar Lösungen für den Konflikt zwischen Geist und Leben, zwischen Vaterwelt und Mutterwelt, zwischen Ehe und Homoerotik, zwischen dem Macht- und Erhöhungstraum einerseits und dem Liebes- und Verschmelzungstraum andererseits anzubieten - und nicht nur Lösungen, sondern rauschhafte Synthesen!» (H. Kurzke: Thomas Mann. Das Leben als Kunstwerk. Eine Biographie, München : C.H. Beck, 1999, p. 237-238).

43. Dans On myself (1940), Thomas Mann décrit ce thème récurrent dans son œuvre comme suit: « es ist die Idee der Heimsuchung, des Einbruchs trunken zerstörender und vernichtender Mächte in ein gefaßtes und mit allen seinen Hoffnungen auf Würde und ein bedingtes Glück der Fassung verschworenes Leben. Das Lied vom errungenen, scheinbar gesicherten Frieden und des den treuen Kunstbau lachend hinfegenden Lebens; von Meisterschaft und Überwältigung, vom Kommen des fremden Gottes war im Anfang, wie es in der Mitte war » (GW XIII, p. 136).

44. «Der Geist, ihr Händereiber, war dem Leben "niemals näher" als eben jetzt, - das Leben selbst sagt es [...]» (Ess I, 208).

45. «Jenes,Wunder der wiedergeborenen Unbefangenheit', auf welches ein wenig später in einem der Dialoge des Autors ausdrücklich und nicht ohne geheimnisvolle Betonung die Rede kam. » (GW VIII, p. 455).

46. « Der geschworene Feind der Triebe, der Leidenschaften [...]» (Ibid., p. 188).

47. Th. Mann: Briefe 1889-1936. Herausgegeben von Erika Mann. Frankfurt/M.: Fischer Taschenbuch Verlag, 1979, p. 214.

48. R. Rolland: Au-dessus de la mêlée, Paris : Ollendorff/Neuchatel : Attinger, 1915, p. 90.

\section{RÉSUMÉS}

Comme de nombreux contemporains, Thomas Mann a accueilli la Première Guerre mondiale avec enthousiasme. Le premier texte inspiré par l'entrée en guerre de l'Allemagne est l'essai Pensées de guerre, rédigé dès août 1914. Thomas Mann y met son art au service de la cause allemande et y défend farouchement le militarisme de l'Allemagne dans lequel il reconnaît une preuve de la grandeur de son pays.

Le discours propagandiste qui parcourt Pensées de guerre cache toutefois un autre discours qui est, lui, esthétique. Les Pensées de guerre se révèlent être, plus profondément, des pensées d'esthète. A travers son engagement pour la guerre, Thomas Mann tente de se positionner en tant qu'artiste par rapport aux nouvelles exigences $\mathrm{du} \mathrm{xx}^{\mathrm{e}}$ siècle et de définir une nouvelle esthétique, notamment autour du concept de Kultur qu'il oppose à celui de Zivilisation - une antithèse que l'on retrouve quelques temps plus tard au centre de la polémique des Considérations d'un apolitique, mettant en avant l'importance de l'irrationnel dans l'art. Paradoxalement, la guerre est supposée aider l'auteur à retrouver le sentiment de vie et d'immédiateté qui manque à l'artiste décadent. L'essai Pensées de guerre montre ainsi que, contrairement à ce que l'on a souvent pensé, il n'existe pas vraiment de rupture avec les œuvres antérieures de l'auteur, mais qu'ici aussi, 
Thomas Mann expérimente avec le surpassement de la décadence et du dilettantisme de la fin de siècle. Si ce texte contient une première prise de position vis-à-vis de la politique et constitue en cela une tentative de se démarquer de l'esthétisme, il reste toutefois un jeu de l'esprit où la guerre sert de prétexte à des considérations artistiques.

Wie viele Zeitgenossen empfängt auch Thomas Mann die Nachricht vom Ersten Weltkrieg mit Enthusiasmus. Das erste Zeugnis seiner Kriegsbegeisterung ist der Aufsatz Gedanken im Kriege, den er gleich nach Ausbruch des Krieges, im August und September 1914, verfaßt. Darin stellt sich der Autor in den Dienst der deutschen Sache und verteidigt u.a. den Militarismus des deutschen Kaiserreiches, in dem er ein Zeichen von kultureller Größe erblickt.

Hinter propagandistischen Aussagen verbirgt sich in Gedanken im Kriege allerdings ein ästhetischer Diskurs. Der Krieg dient Thomas Mann dazu, Stellung zu den gesellschaftlichen und künstlerischen Umwälzungen des beginnenden 20. Jahrhunderts zu beziehen und, insbesondere mit Hilfe des Begriffs der Kultur als Gegensatz zu dem der Zivilisation, eine neue Ästhetik zu definieren, die dem Irrationalen in der Kunst mehr Platz einräumt. Paradoxerweise soll der Krieg dahin führen, die Unmittelbarkeit des Lebens wiederzuerlangen, die dem dekadenten Künstler durch eine zu große Reflexivität abhanden gekommen ist - eine Klage, die beispielsweise eine Novelle wie Tonio Kröger durchzieht. Der Aufsatz Gedanken im Kriege bedeutet deshalb keinen wirklichen Bruch mit den vorangehenden Werken des Autors; er legt vielmehr die Einsicht nahe, daß Thomas Mann auch hier mit der Überwindung der Décadence und des Dilettantismus des fin de siècle experimentiert. Wenn Thomas Mann in diesem Text erstmals politisch Stellung nimmt, und dieser Text somit ein Versuch ist, einer ,lebensdilettantischen' Haltung zu entkommen, bleibt er trotz allem ein unverbindliches Experimentieren, bei dem der Krieg nur ein Vorwand zu künstlerischen Betrachtungen ist.

\section{AUTEUR}

\section{JOËLLE STOUPY}

Université du Littoral-Côte d'Opale (Boulogne-sur-Mer) 